\title{
The prevalence of child sexual abuse in community and student samples: A meta-analysis 岤, 出访
}

\author{
Noemí Pereda ${ }^{\mathrm{a}, *}$, Georgina Guilera ${ }^{\mathrm{b}}$, Maria Forns ${ }^{\mathrm{a}, 1}$, Juana Gómez-Benito ${ }^{\mathrm{b}}$ \\ a Departament de Personalitat, Avaluació i Tractament Psicològics, Facultat de Psicologia, Universitat de Barcelona, Passeig Vall d'Hebron, 171, 08035, Barcelona, Spain \\ b Departament de Metodologia de les Ciències del Comportament, Facultat de Psicologia, Universitat de Barcelona, Passeig Vall d'Hebron, 171, 08035, Barcelona, Spain
}

\section{A R T I C L E I N F O}

\section{Article history:}

Received 17 November 2008

Received in revised form 16 February 2009

Accepted 17 February 2009

\section{Keywords:}

Child sexual abuse

Meta-analysis

Epidemiology

Prevalence

\begin{abstract}
A B S T R A C T
Background: Studies conducted internationally confirm that child sexual abuse is a much more widespread problem than previously thought, with even the lowest prevalence rates including a large number of victims that need to be taken into account.

Objective: To carry out a meta-analysis of the prevalence of child sexual abuse in order to establish an overall international figure.

Methods: Studies were retrieved from various electronic databases. The measure of interest was the prevalence of abuse reported in each article, these values being combined via a random effects model. A detailed analysis was conducted of the effects of various moderator variables.

Results: Sixty-five articles covering 22 countries were included. The analysis showed that $7.9 \%$ of men $(7.4 \%$ without outliers) and $19.7 \%$ of women (19.2\% without outliers) had suffered some form of sexual abuse prior to the age of eighteen.

Conclusions: The results of the present meta-analysis indicate that child sexual abuse is a serious problem in the countries analysed.
\end{abstract}

(c) 2009 Elsevier Ltd. All rights reserved.

\section{Contents}

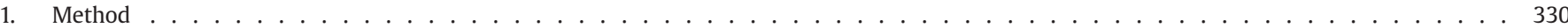

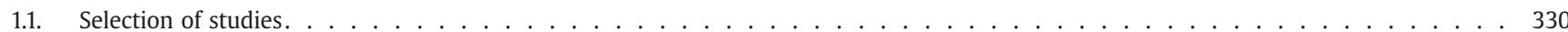

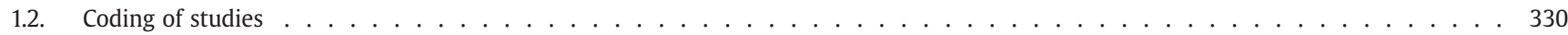

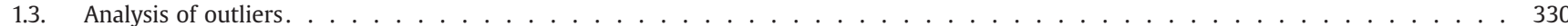

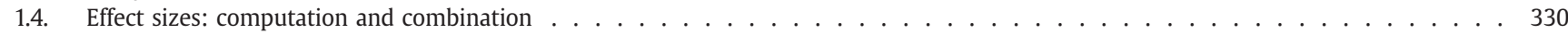

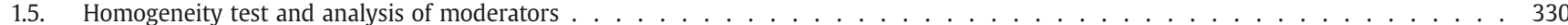

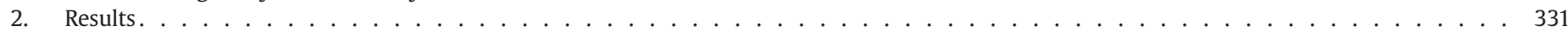

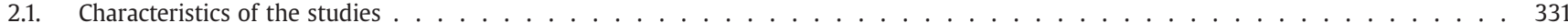

2.2. Analysis of outliers. . . . . . . . . . . . . . . . . . . . . . . . . . . . 331

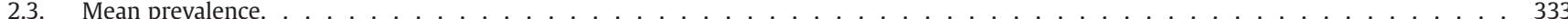

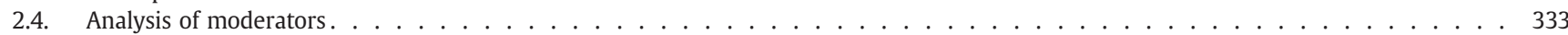

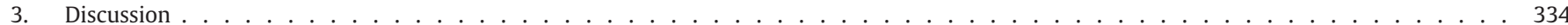

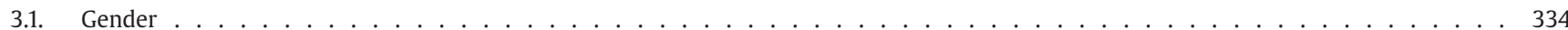

3.2. Geographical and economic area . . . . . . . . . . . . . . . . . . . . . . . . . . . 334

3.3. Criteria for defining sexual abuse and childhood . . . . . . . . . . . . . . . . . . . . . . . . . . 335

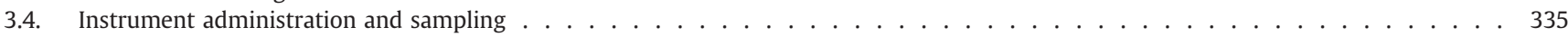

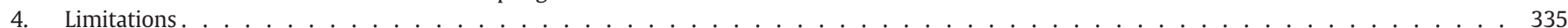

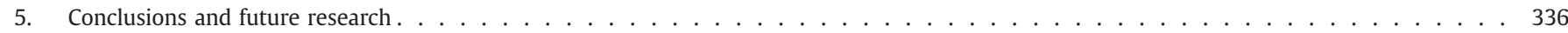

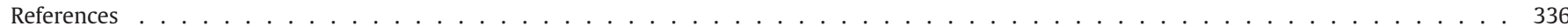

\footnotetext{
is This study was supported in part by grants 2005SGR00365 from the "Departament d'Universitats, Recerca i Societat de la Informació de la Generalitat de Catalunya", and SEJ2005-09144-C02-01-02 from Spain's "Ministerio de Ciencia y Tecnología” under European Regional Development Found (ERDF).

The authors would like to thank all the researchers who kindly provided additional study data upon request. We are indebted to David Gallardo-Pujol for supplying the figures,

* Corresponding author. Tel.: +349331251 13; fax: +34934021362.

E-mail address: npereda@ub.edu (N. Pereda).

1 Tel.: +34933125113; fax: +34934021362.
} 
Child sexual abuse has been considered as one of the most serious public health problems facing society and, above all, children and young people themselves (MacMillan, 1998). Data from published research illustrate that, to a greater or lesser extent, child abuse is a historical constant that occurs in all cultures and societies and at any social level (Walker, Bonner, \& Kaufman, 1988). Child sexual abuse is therefore not an isolated, sporadic or distant reality, but rather a complex and universal problem, one which results from the interaction of individual, family, social and cultural factors (Brown, Cohen, Johnson, \& Salzinger, 1998; Fleming, Mullen, \& Bammer, 1997). The realisation that child sexual abuse was a common form of maltreatment with significant and lasting psychological effects in both the short (Beitchman, Zucker, Hood, DaCosta, \& Akman, 1991; KendallTackett, Meyer, \& Finkelhor, 1993) and long term (Beitchman et al., 1992; Flitter, Elhai, \& Gold, 2003; Jumper, 1995) has led to increasing social and professional interest being shown over the last decade. However, epidemiological studies on this topic remain few and far between and tend to lack methodological rigor; furthermore, most of the research that has been conducted presents conflicting results which, as they cannot be unified, make it impossible to present clear figures regarding the extent of the problem.

Few studies have been published about the incidence of child sexual abuse, whether nationally or internationally, and their number is considerably less than that of prevalence studies regarding the same problem. Incidence refers to the number of cases reported to or detected by the authorities (as hospitals, social services, and the courts) during a given period of time, usually one year (Runyan, 1998; Wynkoop, Capps, \& Priest, 1995). It should be noted that this method of study inevitably underestimates the extent of the problem, and by no means does an incidence rate establish the real occurrence of child sexual abuse (Leventhal, 1998). Factors such as the secrecy which surrounds the abusive situation, the shame felt by the victim when speaking about what has happened, the criminal penalties to which the abuser may be subject, and the young age of victims combined with their dependence upon adults mean that very few victims come forward at the time of abuse, it therefore being highly probable that official statistics underestimate the true extent of the problem (Finkelhor, 1994; Goldman \& Padayachi, 2000; Widom \& Morris, 1997).

Studies about the prevalence of child sexual abuse are more common than those about its incidence, especially since the end of the 1970s and mainly due to research carried out with the US population (Edgardh \& Ormstad, 2000). However, in recent years an increasing number of prevalence studies have been conducted internationally, including research concerning developing countries such as Costa Rica (Krugman, Mata, \& Krugman, 1992), El Salvador (Barthauer \& Leventhal, 1999) or South Africa (Madu \& Peltzer, 2001). Prevalence refers to the number of individuals who suffered some form of abuse during childhood (usually taken as up to age 18, although this criterion varies from study to study) and who are then detected by means of retrospective studies (Runyan, 1998; Wynkoop et al., 1995).

It has been shown that retrospective recall is the most realistic way of approaching the true magnitude of the problem of child sexual abuse (Hardt \& Rutter, 2004). On the other hand, other authors, however, have stated that there might be some difficulties related to the accuracy and feasibility of child self-report (Bruck, \& Ceci, 1999). Obviously, the use of self-report to assess personal experiences such as child sexual abuse, one that is rarely spoken about, increases the risk of possible false negatives and this may be an obstacle in terms of obtaining an accurate estimate (Oates et al., 2000). However, this risk is much greater than the relatively small number of victims who would make false allegations (Fergusson, Horwood, \& Woodward, 2000), and therefore, at all events, prevalence findings would be conservative.

The research carried out to date varies in terms of the procedures used for sample selection, the type of questionnaire or interview employed, the age limit set for classifying subjects as a child victim, and, especially, the criteria used to define whether or not a behaviour should be classified as sexual abuse (Leventhal, 1998). This means that the figures vary enormously from one study to another, and the differences between prevalence rates are considered to be due to methodological differences rather than real differences between study populations (Finkelhor, 1994; Wynkoop et al., 1995).

The importance of sample characteristics and the methodology used to derive prevalence rates has been discussed in several studies, it being highlighted that the percentages vary according to the method through which information was obtained (Starr, Dubowitz, \& Bush, 1990), the context in which questions are asked (Koss, 1993), the number of questions asked (Finkelhor, 1979), the gender of participants (Dhaliwal, Gauzas, Antonowicz, \& Ross, 1996; Romano \& De Luca, 2001) and the ease or difficulty with which participants are able to talk about their sexual experiences, especially as regards sexual abuse, this latter aspect being closely related to cultural variables (Runyan, 1998).

Nevertheless, the studies all agree that child sexual abuse is a much more widespread problem than previously estimated (Tschumper, Narring, Meier, \& Michaud, 1998), and even the lowest prevalence rates include a large number of victims who need to be taken into account (Edgardh \& Ormstad, 2000); indeed, research confirms the importance of the problem among both men and women in all the countries studied (Finkelhor, 1994).

Noteworthy in this regard is the review by Finkelhor (1994) who, after analysing reported prevalence rates for 21 countries, concluded that between 7 and $36 \%$ of women and between 3 and $29 \%$ of men had suffered sexual abuse during childhood. Finkelhor (1994) suggests that establishing the prevalence of sexual abuse in women at around $20 \%$ is a reasonable statistical average, whereas for men the figure would be between 5 and $10 \%$. Obviously, this wide range makes it difficult to determine the true extent of the problem on an international scale. In the same line, a recent study by Pereda, Guilera, Forns, and Gómez-Benito (in press) reviewed the prevalence of sexual abuse in 28 countries and concluded, on the basis of the studies reviewed, that up to $53 \%$ of women and $60 \%$ of men had suffered some form of abuse.

Previous meta-analyses in the child sexual abuse field have mainly examined the psychological impact of this experience (general effects: Jumper, 1995; Neumann, Houskamp, Pollock, \& Briere, 1996; Oddone Paolucci, Genuis, \& Violato, 2001; Rind \& Tromovitch, 1997; Rind, Tromovitch, \& Bauserman, 1998; non-suicidal self-injury: Klonsky \& Moyer, 2008; non-epileptic seizures: Sharpe \& Faye, 2006; chronic pelvic pain: McGowan, Clark-Carter, \& Pitts, 1998; cortisol levels and post-traumatic stress disorder: Meewise, Reitsma, De Vries, Gersons, \& Olff, 2007; Tolin \& Foa, 2006; HIV risk behaviour: Arriola, Louden, Doldren, \& Fortenberry, 2005; eating disorders: Smolak \& Murnen, 2002; borderline personality disorder: Fossati, Madeddu, \& Maffei, 1999; revictimisation: Roodman \& Clum, 2001). Studies have also analysed the social support perceived by the victims (Golding, Wilsnack, \& Cooper, 2002), the types of therapy offered to them (De Jong \& Gorey, 1996; Hetzel-Riggin, Brausch, \& Montgomery, 2007; Peleikis \& Dahl, 2005; Reeker, Ensing, \& Elliot, 1997), the techniques used to assess sexual abuse (West, 1998), and the effectiveness of prevention programmes (Berrick \& Barth, 1992; Davis \& Gidycz, 2000; Rispens, Aleman, \& Goudena, 1997). However, to date no research has sought to integrate the results obtained from epidemiological reviews from a meta-analytic perspective, addressing those variables which could be modulating the prevalence of child sexual abuse.

In this context, the aim of the present study was to conduct a metaanalysis of prevalence studies in the field of sexual abuse, the objectives being twofold: to determine an overall international figure that is able to illustrate the extent of this problem; and to examine the potential moderator variables that may be influencing this prevalence rate. This latter objective is especially important given the variability found in the literature on child sexual abuse as regards, for example, the methodology or definition of abuse used by studies. 


\section{Method}

\subsection{Selection of studies}

The present study included papers published in scientific journals and which met the following inclusion criteria: a) their main or secondary objective was to determine the prevalence of child sexual abuse; b) they used non-clinical samples; c) they reported the prevalence of child sexual abuse separately for men and women; and d) they reported sufficient data to determine the corresponding prevalence and sample size.

Studies were located via the databases Psycinfo, Medline, and Science Citation Index and Social Sciences Citation Index of the Web of Science. The search was based on the following terms: prevalence AND (child sexual abuse OR childhood sexual abuse).

As a complement to the above we conducted a hand search of documents published in specialist journals in the field, specifically in Child Abuse \& Neglect and the Journal of Child Sexual Abuse. We also examined the reference lists of other reviews about the prevalence of child sexual abuse in non-clinical populations (Finkelhor, 1994; Pilkington \& Kremer, 1995; Putnam, 2003), as well as those of published studies in order to obtain additional reports.

In those cases where it was not possible to access a particular report, the corresponding authors were contacted directly in order to request a copy of their article. The only article that we were unable to include, even after contacting the authors, was a paper written in Danish (Larsen \& Helweg-Larsen, 2003).

\subsection{Coding of studies}

The variables taken into account were: a) the authors of the paper; b) the year of publication; c) the country in which the study was conducted; d) this country's level of economic development (developing or developed) according to the World Economic Outlook Database of the International Monetary Fund (2007); e) the continent on which this country is located; f) the gender of the sample studied; g) the mean age of participants (where age was expressed as a range the corresponding mid-point was used); h) the type of sample (general or students); i) the area (local or national); j) the type of sampling (probabilistic or non-probabilistic); k) the method of data collection (self-report questionnaire or interview); l) the definition of sexual abuse (broad - includes non-contact sexual abuse, such as exhibitionism and sexual propositions - or narrow including contact sexual abuse only); and $\mathrm{m}$ ) the age used to define childhood. The prevalence reported by each study was also coded as a measure of interest, as were sample sizes so as to compute the corresponding effect sizes.

In order to avoid dependency effects between studies it was not possible to include more than one prevalence rate per sample; therefore, various decisions had to be made during the coding process as regards the information included in the original studies. Those which used different definitions of sexual abuse (broad and narrow) for the same sample were coded as broad, including a greater variety of types of sexual abuse. Similarly, in those studies that derived prevalence rates for various age-definitions of childhood, the widest age range used was coded. In those studies which made a distinction according to ethnic group, or any other variable not considered in the present analysis, the corresponding prevalence rates were combined in order to obtain a single index of overall prevalence.

These variables were coded by two professionals completely independently of one another. The rate of initial agreement in the coding of prevalence and sample sizes was $95.05 \%$, while the figure for moderator variables was $95.84 \%$. In those few cases where a discrepancy arose this was documented and a consensus was reached.

\subsection{Analysis of outliers}

Outliers were identified graphically using box plots and then eliminated from the calculation of mean prevalence in order to see whether the presence of heterogeneity in the prevalence rates was due to the influence of a few more extreme values. These outliers were also analysed in greater detail so as to identify any characteristics of the study in question which might account for them.

\subsection{Effect sizes: computation and combination}

The measure of interest for each of the studies was the prevalence of child sexual abuse, this being used as a measure of effect size. Thus, from each of the studies a single effect size was derived for women and another for men. In order to avoid bias in the estimates of mean effect sizes the prevalence rate was transformed into a logit event rate and the standard error of this measure was computed. The results were then weighted by the inverse of their variance according to a random effects model (method of moments) so as to give more weight to those studies with more precise measures (Hedges \& Olkin, 1985), in other words, with larger sample sizes. Finally, the logits were once again transformed into proportions in order to present the data in a more comprehensible way.

The choice of the random effects model to combine the studies in the meta-analysis was based on the child sexual abuse literature, which argues that the presence of variability in the prevalence rates reported by different studies may be due to the use of different methodologies (Finkelhor, 1994; Wynkoop et al., 1995). Unlike the fixed effects model, the random effects model presupposes that the studies included present prevalence rates from among all the possible values of prevalence in the population and, therefore, the differences observed are due not simply to sampling error, but rather to some other factor such as the influence of moderator variables. In other words, the random effects model assumes that the population prevalence is an average of all the possible prevalence rates.

\subsection{Homogeneity test and analysis of moderators}

The homogeneity of the studies was tested by means of the $Q$ statistic (Hedges \& Olkin, 1985), which approximates to a Chi-square distribution with $k-1$ degrees of freedom, where $k$ is the number of prevalence rates. Under the null hypothesis of homogeneity the differences between studies can only be attributed to sampling error; in contrast, if the assumption of homogeneity is violated it is usual for the possible sources of variation to be explored by studying moderator variables.

In the present study, when the moderator variable was categorical we applied an analysis of variance for meta-analysis. The between-study homogeneity statistic $\left(Q_{B}\right)$ reflects the amount of heterogeneity that can be attributed to the moderator variable, whereas the within-study homogeneity statistic $\left(Q_{W}\right)$ indicates the degree of heterogeneity that remains within the category in question. In the case of continuous moderator variables we used weighted simple regression (method of moments), where $Q_{R}$ indicates the portion of variability associated with the regression model and $Q_{E}$ represents the variability unaccounted for by the model. In order to determine the combined influence of various moderator variables, and as a complement to the univariate analyses, a weighted multiple regression was computed using the method of moments.

For the computation and combination of effect sizes, and in the analysis of moderator variables, we used the Comprehensive Meta Analysis software (Borenstein \& Rothstein, 1999) and the statistical package SPSS version 15.0.1 (SPSS Inc., 2006). The level of significance was set at .05 . 


\section{Results}

\subsection{Characteristics of the studies}

Of the eligible articles, those which did not meet one or more of the previously-established inclusion criteria were excluded. Two studies were also excluded because they were based on the same data as another publication that had already been included, along with a further two in which it was impossible to distinguish between the prevalence of child sexual abuse and that of sexual abuse in adulthood.

The final sample thus comprised 65 original articles that met the inclusion criteria (see $*$ in the list of references) and which provided information about child sexual abuse in 22 countries. Clearly, the same study may report prevalence rates for both genders and we found that 37 studies included data with male samples, reaching an overall sample size of 37,904 individuals, while 63 worked with female samples, the total sample size in this case being 63,118 women; in overall terms this is equivalent to the inclusion of 100 studies. Of the articles analysed $68.0 \%$ used a broad definition of child sexual abuse, and in $58.5 \%$ of these the age limit for childhood was set at 15,16 or 17 . The majority of articles referred to samples drawn from the general population (50.8\%), used probabilistic designs (61.5\%), recruited samples from local areas (70.8\%), used self-report questionnaires to obtain data (67.7\%), and were carried out in the United States of America $(32.3 \%)$

\subsection{Analysis of outliers}

Two outliers were detected: one among the studies using male samples (i.e. Madu \& Peltzer, 2001) and another with women (i.e. Wyatt, 1985), both of which reported prevalence rates above $60 \%$. In the study by Madu and Peltzer (2001), carried out in South Africa, this high rate of contact sexual abuse among the sample of young men analysed was explained in terms of the particular socio-demographic characteristics of the province in which the research was conducted; specifically, a large number of families in which the paternal figure

Table 1

Main characteristics of the studies and the prevalence rate of child sexual abuse in men.

\begin{tabular}{|c|c|c|c|c|c|c|c|c|c|c|c|c|}
\hline Study name & Country & Development & Population & Age & Area & Sampling & Administration & Definition & $\begin{array}{c}\text { Age } \\
\text { definition }\end{array}$ & $\begin{array}{l}\text { Event } \\
\text { rate }\end{array}$ & $\begin{array}{l}\text { Lower } \\
\text { limit }\end{array}$ & $\begin{array}{l}\text { Upper } \\
\text { limit }\end{array}$ \\
\hline $\begin{array}{l}\text { *Arreola, Neilands, Pollack, Paul, and Catania } \\
\text { (2005) }\end{array}$ & USA & 2 & G & & $\mathrm{L}$ & $\mathrm{P}$ & I & & $<=17$ & 0.103 & 0.092 & 0.115 \\
\hline Baker and Duncan (1985) & $\begin{array}{l}\text { Great } \\
\text { Britain }\end{array}$ & 2 & G & & $\mathrm{N}$ & $\mathrm{P}$ & I & B & $<=15$ & 0.080 & 0.065 & 0.099 \\
\hline Basile, Chen, Black, and Saltzman (2007) & USA & 2 & G & 43 & $\mathrm{~N}$ & $\mathrm{P}$ & I & $\mathrm{N}$ & $<=17$ & 0.015 & 0.012 & 0.018 \\
\hline *Bendixen, Muus, and Schei (1994) & Norway & 2 & $\mathrm{~S}$ & 22.7 & $\mathrm{~L}$ & $\mathrm{P}$ & Q & $\mathrm{B}$ & $<=17$ & 0.035 & 0.022 & 0.056 \\
\hline *Bouvier et al. (1999) & Switzerland & 2 & $\mathrm{~S}$ & 15 & $\mathrm{~L}$ & $\mathrm{P}$ & Q & B & & 0.109 & 0.086 & 0.138 \\
\hline *Briere and Elliott (2003) & USA & 2 & G & 46 & $\mathrm{~N}$ & $\mathrm{P}$ & Q & $\mathrm{N}$ & $<=17$ & 0.142 & 0.113 & 0.177 \\
\hline *Chen, Dunne, and Han (2004) & China & 1 & $\mathrm{~S}$ & 17 & $\mathrm{~L}$ & NP & Q & $\mathrm{B}$ & $<=15$ & 0.105 & 0.089 & 0.124 \\
\hline $\begin{array}{l}\text { Choquet, Darves-Bornoz, Ledoux, } \\
\text { Manfredi, and Hassler (1997) }\end{array}$ & France & 2 & $\mathrm{~S}$ & 16 & $\mathrm{~N}$ & $\mathrm{P}$ & Q & $\mathrm{N}$ & & 0.006 & 0.004 & 0.009 \\
\hline De Paúl, Milner, and Múgica (1995) & Spain & 2 & S & 21 & $\mathrm{~L}$ & NP & Q & B & & 0.097 & 0.053 & 0.170 \\
\hline Edgardh and Ormstad (2000) & Sweden & 2 & Both & 17 & $\mathrm{~N}$ & $P$ & Q & B & & 0.042 & 0.016 & 0.106 \\
\hline *Ernts, Angst, and Földényi (1993) & Switzerland & 2 & G & 29.5 & $\mathrm{~L}$ & $\mathrm{P}$ & I & B & $<=15$ & 0.030 & 0.014 & 0.066 \\
\hline *Figueiredo et al. (2004) & Portugal & 2 & G & 38.6 & $\mathrm{~L}$ & $P$ & Q & B & & 0.026 & 0.014 & 0.048 \\
\hline $\begin{array}{l}\text { *Finkelhor, Hotaling, Lewis, and Smith } \\
\text { (1990) }\end{array}$ & USA & 2 & G & & $\mathrm{N}$ & $\mathrm{P}$ & I & B & $<=18$ & 0.160 & 0.140 & 0.182 \\
\hline *Fritz, Stoll, and Wagner (1981) & USA & 2 & $\mathrm{~S}$ & & $\mathrm{~L}$ & $\mathrm{NP}$ & Q & $\mathrm{N}$ & & 0.048 & 0.031 & 0.073 \\
\hline Goldman 1988 & Australia & 2 & $\mathrm{~S}$ & 22 & $\mathrm{~L}$ & $\mathrm{NP}$ & Q & B & $<=15$ & 0.090 & 0.065 & 0.123 \\
\hline *Goldman and Padayachi (1997) & Australia & 2 & $\mathrm{~S}$ & 21.1 & $\mathrm{~L}$ & $\mathrm{NP}$ & Q & B & $<=16$ & 0.186 & 0.130 & 0.259 \\
\hline *Jumaian (2001) & Jordanian & 1 & $\mathrm{~S}$ & 19 & $\mathrm{~L}$ & NP & Q & $\mathrm{N}$ & $<=13$ & 0.270 & 0.192 & 0.365 \\
\hline Kercher and McShane (1984) & USA & 2 & G & & $\mathrm{L}$ & $\mathrm{P}$ & Q & B & & 0.030 & 0.018 & 0.051 \\
\hline Krugman et al. (1992) & Costa Rica & 1 & $\mathrm{~S}$ & 19.5 & $\mathrm{~L}$ & NP & Q & B & & 0.128 & 0.093 & 0.174 \\
\hline *Lodico, Gruber, and DiClemente (1996) & USA & 2 & $\mathrm{~S}$ & 16 & $\mathrm{~L}$ & $\mathrm{P}$ & Q & $\mathrm{N}$ & & 0.041 & 0.035 & 0.049 \\
\hline $\begin{array}{l}\text { *López, Carpintero, Hernández, Martín, } \\
\text { and Fuertes (1995) }\end{array}$ & Spain & 2 & G & 39 & $\mathrm{~N}$ & $\mathrm{P}$ & I & B & $<=16$ & 0.150 & 0.128 & 0.175 \\
\hline *MacMillan et al. (1997) & Canada & 2 & G & & $\mathrm{N}$ & $\mathrm{P}$ & Q & B & & 0.043 & 0.037 & 0.049 \\
\hline Madu and Peltzer (2001) & $\begin{array}{l}\text { South } \\
\text { Africa }\end{array}$ & 1 & $\mathrm{~S}$ & 18.5 & $\mathrm{~L}$ & $\mathrm{P}$ & Q & $\mathrm{N}$ & $<=16$ & 0.609 & 0.538 & 0.675 \\
\hline *May-Chahal and Cawson (2005) & $\begin{array}{c}\text { Great } \\
\text { Britain }\end{array}$ & 2 & G & 21 & $\mathrm{~N}$ & $\mathrm{P}$ & I & B & $<=15$ & 0.110 & 0.094 & 0.129 \\
\hline *McCrann, Lalor, and Katabaro (2006) & Tanzania & 1 & $\mathrm{~S}$ & 36.5 & $\mathrm{~L}$ & $\mathrm{NP}$ & Q & $\mathrm{N}$ & $<=17$ & 0.250 & 0.203 & 0.304 \\
\hline $\begin{array}{l}\text { *Nelson, Higginson, and Grant-Worley } \\
\quad \text { (1994) }\end{array}$ & USA & 2 & $\mathrm{~S}$ & & $\mathrm{~L}$ & $\mathrm{P}$ & Q & B & & 0.081 & 0.066 & 0.098 \\
\hline *Pereda and Forns (2007) & Spain & 2 & $\mathrm{~S}$ & 22 & $\mathrm{~L}$ & $\mathrm{P}$ & Q & $\mathrm{N}$ & $<=17$ & 0.155 & 0.119 & 0.199 \\
\hline *Priest (1992) & USA & 2 & $\mathrm{~S}$ & 19.92 & $\mathrm{~N}$ & $\mathrm{P}$ & Q & B & $<=16$ & 0.124 & 0.093 & 0.162 \\
\hline *Rew, Esparza, and Sands (1991) & USA & 2 & $\mathrm{~S}$ & 24 & $\mathrm{~L}$ & $\mathrm{P}$ & Q & B & $<=17$ & 0.225 & 0.167 & 0.296 \\
\hline $\begin{array}{l}\text { *Robin, Chester, Rasmussen, Jaranson, } \\
\text { and Goldman (1997) }\end{array}$ & USA & 2 & G & & $\mathrm{L}$ & NP & I & $\mathrm{N}$ & $<=15$ & 0.139 & 0.093 & 0.202 \\
\hline *Ross et al. (2005) & China & 1 & G & 42 & $\mathrm{~L}$ & NP & I & B & & 0.002 & 0.000 & 0.026 \\
\hline *Sariola and Uutela (1994) & Finland & 2 & $S$ & 15.5 & $\mathrm{~N}$ & $\mathrm{P}$ & Q & $\mathrm{N}$ & & 0.040 & 0.034 & 0.047 \\
\hline *Schein et al. (2000) & Israel & 2 & G & 36 & $\mathrm{~N}$ & $\mathrm{P}$ & Q & B & & 0.157 & 0.123 & 0.199 \\
\hline $\begin{array}{l}\text { *Siegel, Sorenson, Golding, Burnam, and } \\
\quad \text { Stein (1987) }\end{array}$ & USA & 2 & G & & $\mathrm{L}$ & $\mathrm{P}$ & I & $\mathrm{N}$ & $<=15$ & 0.038 & 0.029 & 0.049 \\
\hline *Singh, Yiing, and Nurani (1996) & Malaysia & 1 & $\mathrm{~S}$ & 22 & $\mathrm{~L}$ & NP & Q & B & $<=17$ & 0.021 & 0.007 & 0.064 \\
\hline $\begin{array}{l}\text { Sorrenti-Little, Bagley, and Robertson } \\
\text { (1984) }\end{array}$ & Canada & 2 & $\mathrm{~S}$ & & $\mathrm{~L}$ & NP & Q & B & $<=12$ & 0.055 & 0.029 & 0.102 \\
\hline *Tang (2002) & China & 1 & $\mathrm{~S}$ & 21.02 & $\mathrm{~L}$ & $\mathrm{NP}$ & Q & B & $<=16$ & $\begin{array}{l}0.043 \\
0.087\end{array}$ & $\begin{array}{l}0.031 \\
0.083\end{array}$ & $\begin{array}{l}0.059 \\
0.090\end{array}$ \\
\hline
\end{tabular}

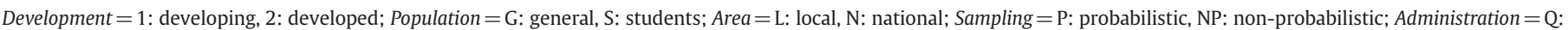
questionnaire, I: interview; Definition = B: broad, N: narrow. 
Table 2

Main characteristics of the studies and the prevalence rate of child sexual abuse in women.

\begin{tabular}{|c|c|c|c|c|c|c|c|c|c|c|c|c|}
\hline Study name & Country & Development & Population & Age & Area & Sampling & Administration & Definition & $\begin{array}{c}\text { Age } \\
\text { definition }\end{array}$ & $\begin{array}{l}\text { Event } \\
\text { rate }\end{array}$ & $\begin{array}{l}\text { Lower } \\
\text { limit }\end{array}$ & $\begin{array}{l}\text { Upper } \\
\text { limit }\end{array}$ \\
\hline *Alami and Kadri (2004) & Morocco & 1 & $\mathrm{G}$ & 36.76 & $\mathrm{~L}$ & $\mathrm{P}$ & Q & B & & 0.092 & 0.073 & 0.115 \\
\hline $\begin{array}{l}\text { Anderson, Martin, Mullen, Romans, and } \\
\text { Herbison (1993) }\end{array}$ & $\begin{array}{c}\text { New } \\
\text { Zealand }\end{array}$ & 2 & G & 41.5 & $\mathrm{~N}$ & $\mathrm{P}$ & Both & B & $<=15$ & 0.319 & 0.279 & 0.361 \\
\hline Arroyo, Simpson, and Aragon (1997) & USA & 2 & $\mathrm{~S}$ & 24.7 & $\mathrm{~L}$ & $\mathrm{NP}$ & Q & $\mathrm{N}$ & $<=15$ & 0.312 & 0.255 & 0.376 \\
\hline *Back et al. (2003) & $\begin{array}{l}\text { Singapore/ } \\
\text { USA }\end{array}$ & 2 & $\mathrm{~S}$ & 19.19 & $\mathrm{~L}$ & NP & Q & B & $<=17$ & 0.159 & 0.096 & 0.251 \\
\hline *Bagley and Ramsay (1986) & Canada & 2 & G & & $\mathrm{L}$ & $\mathrm{P}$ & Q & $\mathrm{N}$ & $<=15$ & 0.217 & 0.178 & 0.261 \\
\hline Baker and Duncan (1985) & $\begin{array}{l}\text { Great } \\
\text { Britain }\end{array}$ & 2 & G & & $\mathrm{N}$ & $\mathrm{P}$ & I & B & $<=15$ & 0.120 & 0.102 & 0.141 \\
\hline Barthauer and Leventhal (1999) & El Salvador & 1 & G & 34.7 & $\mathrm{~L}$ & NP & I & B & $<=17$ & 0.169 & 0.103 & 0.265 \\
\hline Basile et al. (2007) & USA & 2 & G & 43 & $\mathrm{~N}$ & $\mathrm{P}$ & I & $\mathrm{N}$ & $<=17$ & 0.068 & 0.062 & 0.076 \\
\hline *Bendixen et al. (1994) & Norway & 2 & $\mathrm{~S}$ & 22.7 & $\mathrm{~L}$ & $\mathrm{P}$ & Q & B & $<=17$ & 0.194 & 0.162 & 0.231 \\
\hline *Bouvier et al. (1999) & Switzerland & 2 & $\mathrm{~S}$ & 15 & $\mathrm{~L}$ & $\mathrm{P}$ & Q & B & & 0.338 & 0.300 & 0.378 \\
\hline *Briere and Runtz (1988) & USA & 2 & $\mathrm{~S}$ & 19.8 & $\mathrm{~L}$ & $\mathrm{NP}$ & Q & $\mathrm{N}$ & $<=14$ & 0.147 & 0.110 & 0.194 \\
\hline *Briere and Elliott, (2003) & USA & 2 & G & 46 & $\mathrm{~N}$ & $\mathrm{P}$ & $\mathrm{Q}$ & $\mathrm{N}$ & $<=17$ & 0.323 & 0.282 & 0.366 \\
\hline $\begin{array}{l}\text { Bushnell, Wells, and Oakley-Browne } \\
\text { (1992) }\end{array}$ & $\begin{array}{c}\text { New } \\
\text { Zealand }\end{array}$ & 2 & G & 31 & $\mathrm{~L}$ & $\mathrm{NP}$ & I & $\mathrm{N}$ & & 0.130 & 0.096 & 0.173 \\
\hline *Calam and Slade (1989) & $\begin{array}{l}\text { Great } \\
\text { Britain }\end{array}$ & 2 & $\mathrm{~S}$ & 21.33 & $\mathrm{~L}$ & NP & Q & B & $<=13$ & 0.310 & 0.237 & 0.394 \\
\hline${ }^{*}$ Chen et al. (2004) & China & 1 & $\mathrm{~S}$ & 17 & $\mathrm{~L}$ & NP & Q & B & $<=15$ & 0.167 & 0.147 & 0.190 \\
\hline *Chen, Dunne, and Han (2006) & China & 1 & $\mathrm{~S}$ & 19.5 & $\mathrm{~L}$ & NP & Q & B & $<=15$ & 0.219 & 0.179 & 0.266 \\
\hline *Choquet et al. (1997) & France & 2 & $\mathrm{~S}$ & 16 & $\mathrm{~N}$ & $\mathrm{P}$ & Q & $\mathrm{N}$ & & 0.009 & 0.007 & 0.012 \\
\hline *Collings (1997) & $\begin{array}{l}\text { South } \\
\text { Africa }\end{array}$ & 1 & $\mathrm{~S}$ & 19.9 & $\mathrm{~L}$ & NP & Q & $\mathrm{N}$ & $<=17$ & 0.348 & 0.312 & 0.386 \\
\hline *De Paúl et al. (1995) & Spain & 2 & S & 21 & $\mathrm{~L}$ & NP & Q & $\mathrm{B}$ & & 0.148 & 0.112 & 0.193 \\
\hline Edgardh and Ormstad (2000) & Sweden & 2 & Both & 17 & $\mathrm{~N}$ & $\mathrm{P}$ & $\mathrm{Q}$ & B & & 0.281 & 0.206 & 0.370 \\
\hline *Ernts et al. (1993) & Switzerland & 2 & G & 29.5 & $\mathrm{~L}$ & $\mathrm{P}$ & I & B & $<=15$ & 0.112 & 0.077 & 0.160 \\
\hline $\begin{array}{l}\text { Fanslow, Robinson, Crengle, and Perese } \\
\text { (2007) }\end{array}$ & $\begin{array}{l}\text { New } \\
\text { Zealand }\end{array}$ & 2 & G & 41 & $\mathrm{~L}$ & $\mathrm{P}$ & Both & $\mathrm{N}$ & $<=14$ & 0.207 & 0.193 & 0.222 \\
\hline *Figueiredo et al. (2004) & Portugal & 2 & G & 35.8 & $\mathrm{~L}$ & $\mathrm{P}$ & Q & B & & 0.027 & 0.015 & 0.047 \\
\hline *Finkelhor et al. (1990) & USA & 2 & G & & $\mathrm{N}$ & $\mathrm{P}$ & I & B & $<=18$ & 0.270 & 0.248 & 0.293 \\
\hline *Fleming (1997) & Australia & 2 & G & & $\mathrm{N}$ & $\mathrm{P}$ & Q & B & $<=15$ & 0.410 & 0.374 & 0.447 \\
\hline *Fritz, Stoll, and Wagner (1981) & USA & 2 & $\mathrm{~S}$ & & $\mathrm{~L}$ & NP & Q & $\mathrm{N}$ & & 0.077 & 0.057 & 0.103 \\
\hline *Gagnon (1965) & USA & 2 & G & & & & I & B & $<=12$ & 0.278 & 0.253 & 0.304 \\
\hline *Goldman and Goldman (1988) & Australia & 2 & $\mathrm{~S}$ & 22 & $\mathrm{~L}$ & $\mathrm{NP}$ & Q & B & $<=15$ & 0.275 & 0.241 & 0.312 \\
\hline *Goldman and Padayachi (1997) & Australia & 2 & $\mathrm{~S}$ & 21.7 & $\mathrm{~L}$ & NP & $\mathrm{Q}$ & B & $<=16$ & 0.446 & 0.389 & 0.504 \\
\hline *Kenny and McEachern (2000) & USA & 2 & $\mathrm{~S}$ & 37 & $\mathrm{~L}$ & $\mathrm{NP}$ & I & B & $<=17$ & 0.180 & 0.128 & 0.246 \\
\hline Kercher and McShane (1984) & USA & 2 & G & & $\mathrm{L}$ & $\mathrm{P}$ & Q & B & & 0.110 & 0.087 & 0.137 \\
\hline Krugman et al. (1992) & Costa Rica & 1 & $\mathrm{~S}$ & 19.5 & $\mathrm{~L}$ & NP & Q & B & & 0.322 & 0.265 & 0.385 \\
\hline *Levett (1989) & $\begin{array}{l}\text { South } \\
\text { Africa }\end{array}$ & 1 & $\mathrm{~S}$ & 23 & $\mathrm{~L}$ & NP & Q & B & $<=17$ & 0.436 & 0.340 & 0.537 \\
\hline *Lodico et al. (1996) & USA & 2 & $\mathrm{~S}$ & 16 & $\mathrm{~L}$ & $\mathrm{P}$ & Q & $\mathrm{N}$ & & 0.165 & 0.152 & 0.179 \\
\hline *López et al. (1995) & Spain & 2 & G & 39 & $\mathrm{~N}$ & $\mathrm{P}$ & $\mathrm{I}$ & B & $<=16$ & 0.220 & 0.194 & 0.248 \\
\hline *MacMillan et al. (1997) & Canada & 2 & G & & $\mathrm{N}$ & $\mathrm{P}$ & Q & B & & 0.128 & 0.119 & 0.137 \\
\hline Madu and Peltzer (2001) & $\begin{array}{l}\text { South } \\
\text { Africa }\end{array}$ & 1 & $S$ & 18.5 & $\mathrm{~L}$ & $\mathrm{P}$ & Q & $\mathrm{N}$ & $<=16$ & 0.532 & 0.465 & 0.598 \\
\hline *May-Chahal and Cawson (2005) & $\begin{array}{l}\text { Great } \\
\text { Britain }\end{array}$ & 2 & G & 21 & $\mathrm{~N}$ & $\mathrm{P}$ & I & B & $<=15$ & 0.210 & 0.191 & 0.230 \\
\hline *Mazza, Dennerstein, and Ryan (1996) & Australia & 2 & G & & $\mathrm{L}$ & $\mathrm{P}$ & Q & B & $<=15$ & 0.393 & 0.372 & 0.414 \\
\hline *McCrann et al. (2006) & Tanzania & 1 & $\mathrm{~S}$ & 36.5 & $\mathrm{~L}$ & NP & Q & $\mathrm{N}$ & $<=17$ & 0.310 & 0.250 & 0.377 \\
\hline $\begin{array}{l}\text { Mullen, Romans-Clarkson, Walton, and } \\
\text { Herbison, (1988) }\end{array}$ & $\begin{array}{c}\text { New } \\
\text { Zealand }\end{array}$ & 2 & G & & $\mathrm{N}$ & $\mathrm{P}$ & Both & B & $<=12$ & 0.131 & 0.098 & 0.173 \\
\hline *Nelson et al. (1994) & USA & 2 & $\mathrm{~S}$ & & $\mathrm{~L}$ & $\mathrm{P}$ & Q & B & & 0.331 & 0.305 & 0.358 \\
\hline *Niederberger (2002) & Switzerland & 2 & G & 30 & $\mathrm{~L}$ & $\mathrm{P}$ & $\mathrm{I}$ & B & $<=15$ & 0.398 & 0.368 & 0.429 \\
\hline *Oaksford and Frude (2001) & $\begin{array}{c}\text { Great } \\
\text { Britain }\end{array}$ & 2 & $\mathrm{~S}$ & 21 & $\mathrm{~L}$ & NP & Q & $\mathrm{B}$ & $<=15$ & 0.131 & 0.092 & 0.184 \\
\hline *Pereda and Forns (2007) & Spain & 2 & $\mathrm{~S}$ & 22 & $\mathrm{~L}$ & $\mathrm{P}$ & Q & $\mathrm{N}$ & $<=17$ & 0.190 & 0.163 & 0.220 \\
\hline *Priest (1992) & USA & 2 & $\mathrm{~S}$ & 19.92 & $\mathrm{~N}$ & $\mathrm{P}$ & $\mathrm{Q}$ & B & $<=16$ & 0.246 & 0.215 & 0.279 \\
\hline *Rew, Esparza, and Sands (1991) & USA & 2 & $\mathrm{~S}$ & 24 & $\mathrm{~L}$ & $\mathrm{P}$ & $\mathrm{Q}$ & $\mathrm{B}$ & $<=17$ & 0.500 & 0.408 & 0.592 \\
\hline *Robin et al. (1997) & USA & 2 & G & & $\mathrm{L}$ & $\mathrm{NP}$ & I & $\mathrm{N}$ & $<=15$ & 0.493 & 0.427 & 0.559 \\
\hline $\begin{array}{l}\text { *Romero, Wyatt, Loeb, Carmona, and } \\
\text { Solis (1999) }\end{array}$ & USA & 2 & G & 32 & $\mathrm{~L}$ & $\mathrm{P}$ & I & $\mathrm{N}$ & $<=17$ & 0.333 & 0.282 & 0.389 \\
\hline *Ross et al. (2005) & China & 1 & G & 42 & $\mathrm{~L}$ & $\mathrm{NP}$ & I & B & & 0.002 & 0.000 & 0.024 \\
\hline *Russell (1983) & USA & 2 & G & & $\mathrm{L}$ & $\mathrm{P}$ & I & B & $<=17$ & 0.540 & 0.508 & 0.572 \\
\hline *Sariola and Uutela (1994) & Finland & 2 & $\mathrm{~S}$ & 15.5 & $\mathrm{~N}$ & $\mathrm{P}$ & Q & $\mathrm{N}$ & & 0.070 & 0.062 & 0.079 \\
\hline *Sariola and Uutela (1996) & Finland & 2 & $\mathrm{~S}$ & 15.5 & $\mathrm{~N}$ & $\mathrm{P}$ & Q & B & & 0.005 & 0.003 & 0.008 \\
\hline *Schei (1990) & Norway & 2 & G & 34.5 & $\mathrm{~L}$ & $\mathrm{P}$ & I & B & & 0.144 & 0.091 & 0.220 \\
\hline *Schein et al. (2000) & Israel & 2 & G & 36 & $\mathrm{~N}$ & $P$ & $Q$ & B & & 0.307 & 0.273 & 0.343 \\
\hline *Siegel et al. (1987) & USA & 2 & G & & $\mathrm{L}$ & $\mathrm{P}$ & $\mathrm{I}$ & $\mathrm{N}$ & $<=15$ & 0.068 & 0.057 & 0.081 \\
\hline *Singh et al. (1996) & Malaysia & 1 & $\mathrm{~S}$ & 22 & $\mathrm{~L}$ & NP & Q & B & $<=17$ & 0.083 & 0.061 & 0.112 \\
\hline Sorrenti-Little et al. (1984) & Canada & 2 & $\mathrm{~S}$ & & $\mathrm{~L}$ & NP & Q & B & $<=12$ & 0.124 & 0.095 & 0.160 \\
\hline *Tang (2002) & China & 1 & $\mathrm{~S}$ & 21.38 & $\mathrm{~L}$ & NP & $\mathrm{Q}$ & B & $<=16$ & 0.074 & 0.060 & 0.091 \\
\hline Tschumper et al. (1998) & Switzerland & 2 & $\mathrm{~S}$ & 17.5 & $\mathrm{~N}$ & $\mathrm{P}$ & Q & B & & 0.186 & 0.174 & 0.198 \\
\hline *Vogeltanz et al. (1999) & USA & 2 & G & & $\mathrm{N}$ & $\mathrm{P}$ & I & B & $<=17$ & 0.240 & 0.209 & 0.274 \\
\hline
\end{tabular}


Table 2 (continued)

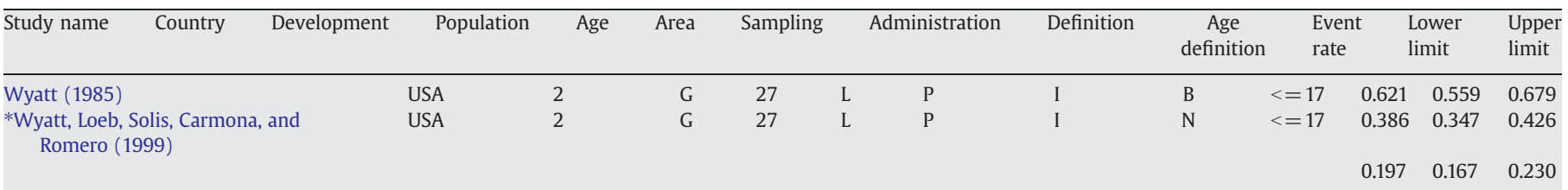

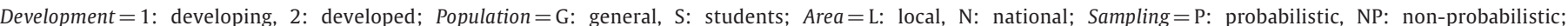
Administration $=\mathrm{Q}$ : questionnaire, I: interview; Definition $=\mathrm{B}$ : broad, N: narrow.

tended to move to other provinces in search of work, thus leaving the way clear for abuse by opportunistic perpetrators and, especially in the case of young men, by female carers. As regards the study by Wyatt (1985), carried out in the United States, the author proposes a number of key factors to bear in mind when interpreting her results, namely the broad definition of sexual abuse (contact and noncontact), the range of the sample across demographic characteristics, and the detailed interview conducted with the women studied, in which sexual abuse was only one of many issues addressed.

\subsection{Mean prevalence}

The mean prevalence of child sexual abuse in males (see Table 1) was $7.9 \%$ (6.0-10.3, 95\% CI), while the figure for women (see Table 2) was $19.7 \%(16.7-23.0,95 \% \mathrm{CI})$. The prevalence of child sexual abuse for females was significantly higher than that for males $\left(Q_{(1)}=35.662\right.$; $p<.05$ ); as significant differences existed between males and females, prevalence rates for sexual abuse are presented separately.

The analysis of homogeneity in the data as regards each of the two genders revealed variability among the studies with men $\left(Q_{(36)}=\right.$ $1582.04 ; p<.05)$ and those with women $\left(Q_{(62)}=4963.40 ; p<.05\right)$.

After eliminating outliers the prevalence of sexual abuse among males was 7.4\% (5.7-9.4, 95\% CI), while the figure for women was $19.2 \%(16.3-22.5,95 \% \mathrm{CI})$, there continued to be heterogeneity between studies.

In fact, much of the literature has already pointed out this feature, there being no standardised practice for determining the prevalence of child sexual abuse, in contrast to the abundance of methodological differences. Consequently, and in line with the second objective of the present study, the next section places greater emphasis on the effect which the various moderator variables may have on reported prevalence rates.

\subsection{Analysis of moderators}

The analyses of moderator variables for men and women are presented in overall terms, since the analyses by gender showed exactly the same effects and there was no interaction between the variable gender and any moderator variable (all $p>.05$ ), the sole exception being the study area (local or national). In this latter case the area of study seems to influence the prevalence of child sexual abuse among women, with the values in local samples $(22.0 \%$; $18.2-26.3$, $95 \% \mathrm{CI}$ ) being higher than in national ones (14.7\%; 10.7-19.8, 95\% CI); however, no such difference was observed with men, for whom the corresponding prevalence rates were $8.6 \%(6.1-12.0,95 \% \mathrm{CI})$ and $6.5 \%$ (4.0-10.5, 95\% CI).

Table 3 shows the individual effects of the different moderator variables considered in the present study, and here it should be noted that the unit of analysis in all cases is the study, not the article. As can be seen, the only variable, apart from gender, with a significant effect on child sexual abuse is the continent on which the study was conducted. Specifically, the highest prevalence rates were found in Africa (34.4\%; $21.1-50.7,95 \% \mathrm{CI}$ ), whereas the lowest were reported in Europe $(9.2 \% ; 6.8-12.3,95 \% \mathrm{CI})$. At all events, after controlling for the effect of this variable there continued to be heterogeneity among the prevalence rates for child sexual abuse within each continent, and therefore caution should be exercised when interpreting these mean values.

As regards the variable country in which the study was conducted the data for men and women are presented separately as an analysis of variance was not possible due to the large number of countries included and the very small number of studies conducted in some of them. This separate presentation also provides information of great importance and interest for the corresponding governments.

As can be seen in Table 4, although there are important differences between countries and between genders as regards reported prevalence rates, several relevant findings can be observed. The South African studies report the highest prevalence rates for both men (60.9\%) and women (43.7\%), whereas the lowest rates correspond to the only study conducted in France, which also concerned both genders ( $0.6 \%$ for males and $0.9 \%$ for females). Jordan presents the second-highest prevalence rate for men (27.0\%), followed by Tanzania (25.0\%). Rates between 10 and $20 \%$ are reported for males in Israel (15.7\%), Spain (13.4\%), Australia (13.0\%) and Costa Rica (12.8\%), while

Table 3

Mean prevalence of child sexual abuse by several moderator variables.

\begin{tabular}{|c|c|c|c|c|c|}
\hline Variable & $k$ & $N$ & Prevalence & $\mathrm{Q}_{\mathrm{B}}$ (d.f.) & $Q_{W}$ (d.f.) \\
\hline Economic development & & & & $0.82(1)$ & \\
\hline Developing & 20 & 8954 & 16.6 & & $852.34(19)^{*}$ \\
\hline Developed & 80 & 92,068 & 13.8 & & $7649.12(79)^{*}$ \\
\hline Continent & & & & $27.70(4)^{*}$ & \\
\hline Africa & 7 & 2357 & 34.4 & & $264.70(6)^{*}$ \\
\hline America & 40 & 47,369 & 15.8 & & $4159.88(39)^{*}$ \\
\hline Asia & 13 & 7110 & 10.1 & & $331.17(12)^{*}$ \\
\hline Europe & 30 & 35,974 & 9.2 & & $2211.53(29)^{*}$ \\
\hline Oceania & 10 & 8212 & 23.9 & & $424.95(9)^{*}$ \\
\hline Population & & & & $0.09(1)$ & \\
\hline Students & 52 & 47,789 & 13.9 & & $3506.32(51)^{*}$ \\
\hline General & 48 & 53,233 & 14.6 & & $4890.52(47)^{*}$ \\
\hline Area & & & & $5.22(1)^{*}$ & \\
\hline Local & 69 & 42,675 & 16.0 & & $4368.32(68)^{*}$ \\
\hline National & 30 & 57,147 & 10.7 & & $3381.41(29)^{*}$ \\
\hline Sampling & & & & $0.39(1)$ & \\
\hline Probabilistic & 63 & 86,894 & 13.6 & & $7477.79(62)^{*}$ \\
\hline Non-probabilistic & 36 & 12,928 & 15.2 & & $956.52(35)^{*}$ \\
\hline Administration & & & & $0.33(1)$ & \\
\hline Questionnaire & 67 & 65,549 & 13.6 & & $5290.92(66)^{*}$ \\
\hline Interview & 30 & 31,807 & 15.1 & & $3114.91(29)^{*}$ \\
\hline Abuse definition & & & & $0.15(1)$ & \\
\hline Broad & 68 & 54,396 & 14.6 & & $4376.89(67)^{*}$ \\
\hline Narrow & 31 & 43,934 & 13.7 & & $3542.14(30)^{*}$ \\
\hline Age abuse definition & & & & $2.28(6)$ & \\
\hline$\leq 12$ & 4 & 2082 & 0.132 & & $78.98(3)^{*}$ \\
\hline$\leq 13$ & 2 & 230 & 0.290 & & $0.44(1)$ \\
\hline$\leq 14$ & 2 & 3593 & 0.176 & & $5.50(1)^{*}$ \\
\hline$\leq 15$ & 22 & 17,566 & 0.170 & & $1611.33(21)^{*}$ \\
\hline$\leq 16$ & 10 & 5730 & 0.217 & & $612.37(9)^{*}$ \\
\hline$\leq 17$ & 25 & 20,496 & 0.226 & & $2442.40(24)^{*}$ \\
\hline$\leq 18$ & 2 & 2626 & 0.210 & & $44.37(1)^{*}$ \\
\hline
\end{tabular}

$k$ : number of studies; $N$ : total simple size; $Q_{\mathrm{B}}$ : between-study homogeneity statistic; $Q_{W}$ : within-study homogeneity statistic.

* $p<.05$. 
Table 4

Mean prevalence of child sexual abuse in men and women according to country of origin.

\begin{tabular}{|c|c|c|c|c|c|}
\hline Country & $k$ & $N$ & Prevalence & Lower limit & Upper limit \\
\hline \multicolumn{6}{|l|}{ Men } \\
\hline Australia & 2 & 528 & 13.0 & 4.9 & 30.1 \\
\hline Canada & 2 & 4683 & 4.8 & 1.7 & 12.9 \\
\hline China & 3 & 2328 & 4.8 & 1.8 & 11.9 \\
\hline Costa Rica & 1 & 268 & 12.8 & 3.2 & 39.3 \\
\hline Finland & 1 & 3322 & .4 .0 & 1.0 & 15.1 \\
\hline France & 1 & 3964 & 0.6 & 0.1 & 2.6 \\
\hline Great Britain & 2 & 2204 & 9.4 & 3.6 & 22.5 \\
\hline Israel & 1 & 350 & 15.7 & 4.1 & 44.8 \\
\hline Jordanian & 1 & 100 & 27.0 & 7.6 & 62.6 \\
\hline Malaysia & 1 & 141 & 2.1 & 0.3 & 12.0 \\
\hline Norway & 1 & 486 & 3.5 & 0.8 & 14.2 \\
\hline Portugal & 1 & 388 & 2.6 & 0.6 & 11.4 \\
\hline South Africa & 1 & 193 & 60.9 & 26.4 & 87.1 \\
\hline Spain & 3 & 1315 & 13.4 & 6.1 & 26.8 \\
\hline Sweden & 1 & 96 & 4.2 & 0.7 & 20.1 \\
\hline Switzerland & 2 & 745 & 6.3 & 2.2 & 16.8 \\
\hline Tanzania & 1 & 282 & 25.0 & 7.1 & 59.1 \\
\hline USA & 11 & 16,511 & 7.5 & 5.1 & 11.1 \\
\hline \multicolumn{6}{|l|}{ Women } \\
\hline Australia & 4 & 3717 & 37.8 & 22.9 & 55.5 \\
\hline Canada & 3 & 6215 & 15.2 & 7.2 & 29.2 \\
\hline China & 4 & 2977 & 10.8 & 5.2 & 21.3 \\
\hline Costa Rica & 1 & 229 & 32.2 & 10.0 & 66.9 \\
\hline El Salvador & 1 & 83 & 16.9 & 4.2 & 48.5 \\
\hline Finland & 2 & 7360 & 2.0 & 0.7 & 5.5 \\
\hline France & 1 & 4176 & 0.9 & 0.2 & 3.8 \\
\hline Great Britain & 4 & 3027 & 18.2 & 9.7 & 31.5 \\
\hline Israel & 1 & 655 & 30.7 & 9.6 & 65.0 \\
\hline Malaysia & 1 & 471 & 8.3 & 2.1 & 28.1 \\
\hline Morocco & 1 & 728 & 9.2 & 2.3 & 30.1 \\
\hline New Zealand & 4 & 3967 & 18.7 & 10.1 & 32.2 \\
\hline Norway & 2 & 628 & 16.9 & 6.7 & 36.6 \\
\hline Portugal & 1 & 447 & 2.7 & 0.6 & 11.4 \\
\hline Singapore & 1 & 88 & 15.9 & 3.9 & 46.7 \\
\hline South Africa & 3 & 950 & 43.7 & 25.1 & 64.2 \\
\hline Spain & 3 & 1937 & 18.5 & 9.0 & 34.2 \\
\hline Sweden & 1 & 114 & 28.1 & 8.2 & 63.2 \\
\hline Switzerland & 4 & 5765 & 24.2 & 13.4 & 39.6 \\
\hline Tanzania & 1 & 204 & 31.0 & 9.5 & 65.8 \\
\hline USA & 19 & 19,380 & 25.3 & 19.7 & 31.8 \\
\hline
\end{tabular}

$k$ : number of studies; $N$ : total simple size.

the remaining countries all have prevalence rates below $10 \%$. In the case of women, Australia (37.8\%), Costa Rica (32.2\%), Tanzania (31.0\%), Israel (30.7\%), Sweden (28.1\%), the United States $(25.3 \%)$ and Switzerland (24.2\%) all report prevalence rates are above $20 \%$. The figures for New Zealand (18.7\%), Spain (18.5\%), Great Britain (18.2\%), El Salvador and Norway (16.9\%), Singapore (15.9\%), Canada (15.2\%) and China (10.8\%) fall between 10 and 20\%. The remaining countries have prevalence rates below $10 \%$.

As regards the mean age of the sample the simple regression for meta-analysis revealed no relationship between this variable and the corresponding prevalence rates $\left(Q_{R(1)}=0.03 ; p=.86\right)$.

Given the complexity of interpreting the univariate results, which showed that none of the moderator variables alone can explain the observed variability in prevalence rates of child sexual abuse, we performed a weighted multiple regression in order to explore which variables made the greatest contribution in terms of explaining the variability in the corresponding prevalence rates.

The two variables entered into the model were gender and area from which the sample was drawn, which together accounted for $19 \%$ of the total observed variability $\left(\beta_{\text {(gender) }}=0.40 ; \beta_{\text {(area) }}=-0.15 ; R^{2}=18.74\right)$. This model was moderately effective in accounting for the variability in prevalence rates $\left(Q_{R(1)}=39.86 ; p<.05\right)$; however, the residual of the model was also statistically significant $\left(Q_{\mathrm{E}(96)}=172.80 ; p<.05\right)$, thus indicating that there continues to be a considerable amount of variability in the data that is not explained by the variables analysed.

\section{Discussion}

The results obtained in the present study confirm that the experience of child sexual abuse is a problem of considerable magnitude in all the societies analysed. Although the epidemiological data from the studies reviewed do not enable definitive prevalence rates to be established this fact should not be used to underestimate the scale of the problem revealed by the data presented here. The results of the present meta-analysis, the first to be carried out with respect to the epidemiology of this problem, are derived from 100 studies and involved sample sizes ranging from 83 to 5434 .

On an international level, $7.9 \%$ of men $(7.4 \%$ if outliers are eliminated) and $19.7 \%$ of women (19.2\% if outliers are eliminated) have suffered some form of sexual abuse prior to the age of eighteen.

\subsection{Gender}

The higher prevalence rates reported among women by the studies reviewed enables us to infer that most victims of child sexual abuse are female, although the important percentage of male victims should not be overlooked (Dhaliwal et al., 1996), especially as the latter phenomenon often goes largely unrecognised and is rarely addressed (Holmes \& Slap, 1998). The ratio for the prevalence of child sexual abuse is usually around one man to every three women (Finkelhor, 1994), this being consistent with the present findings, where the ratio was 2.5 women for each male victim of sexual abuse.

Only one of the studies reviewed (Madu \& Peltzer, 2001) reported a higher number of male victims. However, as the authors themselves point out, this difference could be due to the characteristics of the area of study, since this finding is not replicated in any of the other published studies concerning South Africa.

The comparatively low prevalence rates for child sexual abuse of males has been discussed by several authors (Dhaliwal et al., 1996; Romano \& De Luca, 2001; Violato \& Genuis, 1993), who consider that one of the most important problems is the methodology used in many studies; it is argued that either the experiences of men are not sufficiently captured by the definition of sexual abuse used, or that men fail to identify themselves with certain questions asked. Social attitudes toward sex and stereotypes about the roles of men and women may also prevent men from explaining their experiences, or even mean that they do not regard what happened as sexual abuse (Violato \& Genuis, 1993; Widom \& Morris, 1997). Indeed, it would seem that sex stereotypes present in society can lead men to consider that maintaining sexual relationships with adults, particularly with women, is a way of demonstrating their manliness or masculinity (Coxell, King, Mezey, \& Gordon, 1999).

Another explanation for the lower prevalence rates among men is the possibility of greater shame and the fear that they will be labelled as homosexual (if the aggressor was another man) or weak (if the aggressor was a woman), which may combine with the fact that they are more often accused of having provoked the abuse (Holmes \& Slap, 1998; Romano \& De Luca, 2001).

These factors would seem to influence the low rates of positive responses given by men in studies of child sexual abuse, this being a finding reported by most authors (Dhaliwal et al., 1996; Holmes \& Slap, 1998; Romano \& De Luca, 2001; Violato \& Genuis, 1993).

\subsection{Geographical and economic area}

As regards the continent from which study samples were drawn the highest prevalence rate of child sexual abuse is found in Africa (34.4\%; 21.1-50.7, 95\% CI), represented by Morocco, Tanzania and, 
mainly, South Africa. Europe, including many different countries (e.g. Spain, Finland), shows the lowest prevalence rate (9.2\%; 6.8-12.3, 95\% $\mathrm{CI})$. Finally, America, Asia and Oceania have prevalence rates between 10.1 and $23.9 \%$.

According to the data obtained the continent from which the study sample is drawn is one of the moderator variables that influence the observed differences in prevalence rates. One initial explanation for this, which should not be overlooked, is that there are real differences in the rate of child sexual abuse between one continent and another. Lalor (2004) recently reported a review of various studies, published locally, about the prevalence of child sexual abuse in Tanzania and Kenya. Although the barely controlled methodology used in these studies prevents any quantitative conclusions from being drawn Lalor (2004) suggests that child sexual abuse has increased in these countries for a variety of possible reasons, especially the following: (a) the widespread belief that having sexual relationships with virgins or very young girls is a cure for the human immunodeficiency virus (HIV) and other sexually-transmitted diseases; (b) the loss of traditional values based on bringing up children within the community, which enabled neighbours to observe and correct any maltreatment or abuse on the part of parents; (c) the influence of other cultures, especially as regards sex tourism; (d) poverty, which encourages the youngest girls to prostitute themselves and the parents to support this decision; and (e) the position of women in these societies, where they are always inferior to men and much less valued than in the West. However, a second explanation, which takes account of psychological and cultural aspects of the individuals studied, is that on certain continents there is an increased awareness or ease as regards speaking about sexual experiences, including those identified as sexual abuse (Runyan, 1998).

Thus, the different prevalence rates observed in the present study between continents could indicate that certain societies are characterised by greater social acceptance of the problem, thus helping victims to come forward about their experiences of this kind. Alternatively, these differences could account for very real differences in the occurrence of child sexual abuse. The characteristics of the present study do not enable a single explanation to be established.

Significant differences were also observed in the prevalence rates of child sexual abuse according to the area (national or local) from which the study sample was obtained. Indeed, the existence of sexual abuse appears to be related to the area evaluated, as greater prevalence is found in certain areas. A more exhaustive and detailed study of the effect of country of origin on the prevalence of sexual abuse could facilitate a greater understanding of these results.

Additionally, the results obtained in previous studies have found no - or only a weak - relationship between the experience of sexual abuse and the social class or degree of poverty of the victim's family of origin (Runyan, 1998). This finding would seem to be confirmed when taking into account the general economic status of the sample's country of origin, as is suggested in the present meta-analysis.

\subsection{Criteria for defining sexual abuse and childhood}

In line with the published literature the present paper shows that the definition of sexual abuse and the criteria for classifying the victim as a child differ from study to study. Previous reports have argued that the use of broad (as opposed to narrow) definitions and a cut-off point of 18 to define childhood (rather than younger ages) gives rise to higher prevalence rates (Wynkoop et al., 1995), although the metaanalytic findings do not appear to support this view.

The present results suggest that the definition of child sexual abuse (broad or narrow) used by a study does not influence the different prevalence rates found. This finding is contrary to the reports of other authors who have placed great importance on the definition of sexual abuse as a determining factor in the variability of prevalence rates (Goldman \& Padayachi, 2000; Gorey \& Leslie, 1997; Wynkoop et al.,
1995). However, it should be borne in mind that the fact that one of the two definitions (broad) includes behaviour that is characteristic of the other (narrow) could account for this.

On a related matter we found no significant differences in prevalence rates according to the cut-off age used to define childhood. This finding could be due to the fact that the age at which most child sexual abuse takes place is generally during the pre-pubertal years (that is, between 8 and 12, according to Coleman \& Coleman, 2002). Therefore, sexual abuse would have already occurred regardless of the cut-off age used in the study provided that it was set at older than 12.

\subsection{Instrument administration and sampling}

Methodological differences between studies, including instrument administration and sampling design, have traditionally been considered as one of the variables which might explain the discrepancies among reported prevalence rates (Wynkoop et al., 1995).

The mode of data collection (interview versus self-administered questionnaires) has also been regarded as a variable to take into account when establishing the prevalence rate of sexual abuse (Goldman \& Padayachi, 2000), although the results of the present meta-analysis suggest it does not have a significant effect.

Another issue addressed in the literature has been the reporting of lower prevalence rates in studies where the sample consisted of college students, although there seem to be no clear trends in this regard (Goldman \& Padayachi, 2000). Some studies have provided evidence in support of this idea (Baker \& Duncan, 1985; Kercher \& McShane, 1984; Mullen, Romans-Clarkson, Walton, \& Herbison, 1988), while others have failed to confirm that the prevalence of sexual abuse is lower in this group of subjects (Rind et al., 1998). The findings of the present study are consistent with this latter view.

As regards the mean age of the samples analysed the present study found no evidence to suggest that the age of the sample at the time of study has an influence on the reported prevalence of sexual abuse, there being no significant differences in this respect. Although it has been argued that the use of young samples avoids, at least partially, the memory distortions and problems that might be present with older adults (Halperin et al., 1996) it seems that most victims, regardless of their age when surveyed, would remember the experience of sexual abuse and be able to reveal it in retrospective studies (Pope \& Hudson, 1995).

\section{Limitations}

The present study has several limitations. According to Finkelhor (1993) one of the main problems faced by retrospective studies is that since they are conducted with adults and their results cannot be generalised to the current population of children, for whom the indices and circumstances of victimisation may be very different.

The use of retrospective studies also runs the risk of underestimating the number of real cases of sexual abuse, since in this type of research the percentage of false negatives is higher than that of false positives. These studies are based on the retrospective recall of participants, which may be subject to errors such as the distorted perception of what happened, the reinterpretation of the experience of abuse with the passage of time, complete amnesia due to denial and dissociation from what occurred, or even the conscious desire not to respond sincerely to the questionnaire items (Briere, 1992; Goldman \& Padayachi, 2000; McMillen \& Zuravin, 1998; Prescott et al., 2000).

However, although the value of retrospective studies may be undermined by distorted recall they are often the only method available to many researchers due to the important legal and ethical restrictions applied to cross-sectional or prospective studies with children (Anderson, Martin, Mullen, Romans, \& Herbison, 1993). 
On the other hand, given that prevalence studies are descriptive and do not, therefore, involve statistical significance, the issue of publication bias should not, in theory, be a limitation of the present study. However, it could be that some studies with small samples are less likely to be published, while another issue is that the clinical and social repercussions of child sexual abuse may conflict with political or governmental interests and thus restrict the publication of certain studies or reports.

\section{Conclusions and future research}

Despite the limitations described above, the results of the present meta-analysis remain highly relevant since they indicate that child sexual abuse is a serious problem in each of the countries analysed, this being consistent with the findings of previous reviews (Finkelhor, 1994; Pereda et al., in press).

Although the potential moderator variables do not, on their own, explain the total variability observed in prevalence rates they do reveal certain aspects of relevance as regards the wide range of methodologies used. One way of testing the effect of moderator variables on prevalence rates would be to assign the individuals under study to different levels of the variables (e.g. broad definition versus narrow definition) and to observe any corresponding changes in the rates of child sexual abuse. Although this type of study would require a significant investment of human and financial resources, as well as time, it would contribute to a greater understanding of the variables that affect the prevalence rates of child sexual abuse. Of course, it is also possible that other variables, frequently overlooked in the original articles, are responsible for modulating the prevalence rates of sexual abuse (e.g., characteristics of the family of origin, age at which the sexual abuse began, or the type of aggressor).

Another possibility is that the questions used to obtain information about child sexual abuse do not capture variables of fundamental importance in the explanatory sense. In this context it would be advisable to use qualitative sampling techniques (such as focus groups or cognitive interviews) that would enable exploration of the full range of variables relevant to the problem of child sexual abuse. Cognitive interviewing entails administering questionnaire or test items while collecting additional verbal information about the questionnaire responses, which is used to evaluate the quality of the response or to determine whether the question is generating the information that its author intends (Beatty, 2004).

On the basis of the findings produced through these new techniques, and with the aim of obtaining more comparable results, it would then be useful to develop a research protocol that was not biased by cultural variables or gender. This would require preliminary conceptual work since present formulations do not appear to adequately capture the experience of male children and youth. It would be necessary to formulate specific questions for each sex, since as several authors have pointed out, some questions about child sexual abuse seem not to include men's experience (Dhaliwal et al., 1996; Romano \& De Luca, 2001; Violato \& Genuis, 1993).

The present study found no significant differences in prevalence rates for most of the moderator variables, and it can therefore be concluded that the findings regarding the experience of sexual abuse in childhood are similar when methodological, social and demographic variables are controlled for.

Given that child sexual abuse is, in most cases, a traumatic experience that interferes with the victim's development and has negative repercussions for his/her physical and psychological wellbeing in both the short term (Beitchman et al., 1991; Kendall-Tackett et al., 1993) and the long term (Beitchman et al., 1992; Flitter et al., 2003; Jumper, 1995), there is a need for unbiased prevalence rates that enable each country to develop suitable health and preventive strategies.

\section{References $^{2}$}

*Alami, M., \& Kadri, N. (2004). Moroccan women with a history of child sexual abuse and its long-term repercussions: A population-based epidemiological study. Archives of Women's Mental Health, 7, 237-242.

*Anderson, J., Martin, J., Mullen, P., Romans, S., \& Herbison, P. (1993). Prevalence of childhood sexual abuse experiences in a community sample of women. Journal of the American Academy of Child and Adolescent Psychiatry, 32(5), 911 -919.

*Arreola, S. G., Neilands, T. B., Pollack, L. M., Paul, J. P., \& Catania, J. A. (2005). Higher prevalence of childhood sexual abuse among Latino men who have sex with men than non-Latino men who have sex with men: data from the Urban Men's Health Study. Child Abuse E' Neglect, 29, 285-290.

Arriola, K. R. J., Louden, T., Doldren, M. A., \& Fortenberry, R. M. (2005). A meta-analysis of the relationship of child sexual abuse to HIV risk behaviour among women. Child Abuse \& Neglect, 29(6), 725-746.

*Arroyo, J. A., Simpson, T. L., \& Aragon, A. S. (1997). Childhood sexual abuse among Hispanic and Non-Hispanic white college women. Hispanic Journal of Behavioral Sciences, 19(1), 57-68.

*Back, S. E., Jackson, J. L., Fitzgerald, M., Shaffer, A., Salstrom, S., \& Osman, M. M. (2003). Child sexual and physical abuse among college students in Singapore and the United States. Child Abuse \& Neglect, 27, 1259-1275.

*Bagley, C., \& Ramsay, R. (1986). Sexual abuse in childhood: Psychosocial outcomes and implications for social work practice. Social Work Practice in Sexual Problems, 4, 33-47.

*Baker, A. W., \& Duncan, S. P. (1985). Child sexual abuse: A study of prevalence in Great Britain. Child Abuse \& Neglect, 9, 457-467.

*Barthauer, L. M., \& Leventhal, J. M. (1999). Prevalence and effects of child sexual abuse in a poor, rural community in El Salvador: A retrospective study of women after 12 years of civil war. Child Abuse \& Neglect, 23(11), 1117-1126.

*Basile, K. C., Chen, J., Black, M. C., \& Saltzman, L. E. (2007). Prevalence and characteristics of sexual violence victimization among U.S. adults, 2001-2003. Violence and Victims, 22(4), 437-448.

Beatty, P. (2004). The dynamics of cognitive interviewing. In S. Presser, J. M. Rothgeb, M. P. Couper, J. T. Lessler, E. Martin, J. Martin, \& E. Singer (Eds.), Methods for testing and evaluating survey questionnaires (pp. 45-66). Hoboken, NJ: John Wiley and Sons.

Beitchman, J. H., Zucker, K. J., Hood, J. E., DaCosta, G. A., \& Akman, D. (1991). A review of the short-term effects of child sexual abuse. Child Abuse E Neglect, 15, 537-556.

Beitchman, J. H., Zucker, K. J., Hood, J. E., DaCosta, G. A., Akman, D., \& Cassavia, E. (1992). A review of the long-term effects of child sexual abuse. Child Abuse $\mathcal{E}$ Neglect, 16, 101-118.

*Bendixen, M., Muus, K., \& Schei, B. (1994). The impact of child sexual abuse. A study of a random sample of Norwegian students. Child Abuse \& Neglect, 18, 837-847.

Berrick, J. D., \& Barth, R. P. (1992). Child sexual abuse prevention: Research review and recommendations. Social Work Research E Abstracts, 28(4), 6-15.

Borenstein, M., \& Rothstein, H. (1999). Comprehensive meta-analysis: A computer program for research synthesis. New Jersey: Biostat.

*Bouvier, P., Halpérin, D. S., Rey, H., Jaffé, P. D., Laederach, J., Mounoud, R. -L., et al. (1999). Typology and correlates of sexual abuse in children and youth: Multivariate analyses in a prevalence study in Geneva. Child Abuse E Neglect, 23(8), 779-790.

Briere, J. (1992). Methodological issues in the study of sexual abuse effects. Journal of Consulting and Clinical Psychology, 60(2), 196-203.

*Briere, J., \& Elliott, D. M. (2003). Prevalence and psychological sequelae of self-reported childhood physical and sexual abuse in a general population sample of men and women. Child Abuse \& Neglect, 27, 1205-1222.

*Briere, J., \& Runtz, M. (1988). Symptomatology associated with childhood sexua victimization in a nonclinical adult sample. Child Abuse E' Neglect, 12, 51-59.

Brown, J., Cohen, P., Johnson, J., \& Salzinger, S. (1998). A longitudinal analysis of risk factors for child maltreatment: Findings of a 17-year prospective study of officially recorded and self-reported child abuse and neglect. Child Abuse \& Neglect, 22(11), $1065-1078$.

Bruck, M., \& Ceci, S. J. (1999). The suggestibility of children's memory. Annual Review of Psychology, 50, 419-439.

*Bushnell, J. A., Wells, J. E., \& Oakley-Browne, M. A. (1992). Long-term effects of intrafamilia sexual abuse in childhood. Acta Psychiatrica Scandinavica, 85, 136-142.

*Calam, R. M., \& Slade, P. D. (1989). Sexual experience and eating problems in female undergraduates. International Journal of Eating Disorders, 8(4), 391-397.

*Chen, J., Dunne, M. P., \& Han, P. (2004). Child sexual abuse in China: A study of adolescents in four provinces. Child Abuse \& Neglect, 28, 1171-1186.

*Chen, J., Dunne, M. P., \& Han, P. (2006). Child sexual abuse in Henan province, China: Associations with sadness, suicidality, and risk behaviours among adolescent girls. Journal of Adolescent Health, 38, 544-549.

*Choquet, M., Darves-Bornoz, J. -M., Ledoux, S., Manfredi, R., \& Hassler, C. (1997) Self-reported health and behavioural problems among adolescent victims of rape in France: Results of a cross-sectional survey. Child Abuse E Neglect, 21(9), $823-832$.

Coleman, L., \& Coleman, J. (2002). The measurement of puberty: A review. Journal of Adolescence, 25, 535-550.

*Collings, S. J. (1997). Child sexual abuse in a sample of South African women students: Prevalence, characteristics, and long-term effects. South African Journal of Psychology, 27(1), 37-42.

Coxell, A., King, M., Mezey, G., \& Gordon, D. (1999). Lifetime prevalence, characteristics, and associated problems of non-consensual sex in men: Cross sectional study. British Medical Journal, 318, 846-850.

\footnotetext{
${ }^{2}$ Articles marked with an asterisk were included in the meta-analysis (may not have been cited in the text).
} 
Davis, M. K., \& Gidycz, C. A. (2000). Child sexual abuse prevention programs: A metaanalysis. Journal of Clinical Child Psychology, 29(2), 257-265.

De Jong, T. L., \& Gorey, K. M. (1996). Short-term versus long-term group work with female survivors of childhood sexual abuse: A brief meta-analytic review. Social Work with Groups, 19(1), 19-27.

*De Paúl, J., Milner, J. S., \& Múgica, P. (1995). Childhood maltreatment, childhood social support, and child abuse potential in a Basque sample. Child Abuse E Neglect, 19(8), 907-920.

Dhaliwal, G. K., Gauzas, L., Antonowicz, D. H., \& Ross, R. R. (1996). Adult male survivors of childhood sexual abuse, prevalence, sexual abuse characteristics, and long-term effects. Clinical Psychology Review, 16(7), 619-639.

*Edgardh, K., \& Ormstad, K. (2000). Prevalence and characteristics of sexual abuse in a national sample of Swedish seventeen-year-old boys and girls. Acta Paediatrica, 88, $310-319$.

*Ernts, C., Angst, J., \& Földényi, M. (1993). The Zurich study: XVII. Sexual abuse in childhood: Frequency and relevance for adult morbidity data of a longitudinal epidemiological study. European Archives of Psychiatry and Clinical Neuroscience, 243(3-4), 179-188.

*Fanslow, J. L., Robinson, E. M., Crengle, S., \& Perese, L. (2007). Prevalence of child sexual abuse reported by a cross-sectional sample of New Zealand women. Child Abuse $\mathcal{E}$ Neglect, 31, 935-945.

Fergusson, D. M., Horwood, J. L., \& Woodward, L. J. (2000). The stability of child abuse reports: A longitudinal study of the reporting behaviour of young adults. Psychological Medicine, 30, 529-544

*Figueiredo, B., Bifulco, A., Paiva, C., Maia, A., Fernandes, E., \& Matos, R. (2004). History of childhood abuse in Portuguese parents. Child Abuse \& Neglect, 28, 669-682.

Finkelhor, D. (1979). Sexually victimized children. New York: Free press.

Finkelhor, D. (1993). Epidemiological factors in the clinical identification of child sexual abuse. Child Abuse \& Neglect, 17, 67-70.

Finkelhor, D. (1994). The international epidemiology of child sexual abuse. Child Abuse E' Neglect, 18(5), 409-417.

*Finkelhor, D., Hotaling, G., Lewis, I. A., \& Smith, C. (1990). Sexual abuse in a national survey of adult men and women: Prevalence, characteristics, and risk factors. Child Abuse $\mathcal{E}$ Neglect, $14,19-28$

*Fleming, J. M. (1997). Prevalence of childhood sexual abuse in a community sample of Australian women. Medical Journal of Australia, 166, 65-68.

Fleming, J., Mullen, P., \& Bammer, G. (1997). A study of potential risk factors for sexua abuse in childhood. Child Abuse \& Neglect, 21(1), 49-58.

Flitter, J. M. K., Elhai, J. D., \& Gold, S. N. (2003). MMPI-2 F Scale elevations in adult victims of child sexual abuse. Journal of Traumatic Stress, 16(3), 269-274.

Fossati, A., Madeddu, F., \& Maffei, C. (1999). Borderline personality disorder and childhood sexual abuse: A meta-analytic study. Journal of Personality Disorders 13(3), 268-280.

*Fritz, G. S., Stoll, K., \& Wagner, N. N. (1981). A comparison of males and females who were sexually molested as children. Journal of Sex $\mathcal{E}^{\prime}$ Marital Therapy, 7(1), 54-59.

*Gagnon, J. H. (1965). Female child victims of sex offenses. Social Problems, 13(2), 176-192.

Golding, J. M., Wilsnack, S. C., \& Cooper, M. L. (2002). Sexual assault history and social support: Six general population studies. Journal of Traumatic Stress, 15(3), 187-197.

*Goldman, R. J., \& Goldman, D. G. (1988). The prevalence and nature of child sexual abuse in Australia. American Journal of Sex, Marriage E Family, 9(2), 94-106.

*Goldman, J. D. G., \& Padayachi, U. K. (1997). The prevalence and nature of child sexua abuse in Queensland, Australia. Child Abuse E Neglect, 21(5), 489-498.

Goldman, J. D. G., \& Padayachi, U. K. (2000). Some methodological problems in estimating incidence and prevalence in child sexual abuse research. The Journal of Sex Research, 37(4), 305-314.

Gorey, K. M., \& Leslie, D. R. (1997). The prevalence of child sexual abuse: Integrative review adjustment for potential response and measurement biases. Child Abuse $\varepsilon$ Neglect, 21(4), 391-398.

Halperin, D. S., Bouvier, P., Jaffe, P. D., Mounoud, R. L., Pawlak, C. H., Laederach, J., et al (1996). Prevalence of child sexual abuse among adolescents in Geneva: Results of cross sectional survey. British Medical Journal, 312(7042), 1326-1329.

Hardt, J., \& Rutter, M. (2004). Validity of adult retrospective reports of adverse childhood experiences: Review of the evidence. Journal of Child Psychology and Psychiatry, 45(2), $260-273$.

Hedges, L. V., \& Olkin, I. (1985). Statistical methods for meta-analysis. Orlando: Academic Press

Hetzel-Riggin, M. D., Brausch, A. M., \& Montgomery, B. S. (2007). A meta-analytic investigation of therapy modality outcomes for sexually abused children and adolescents: An exploratory study. Child Abuse E' Neglect, 31(2), 125-141.

Holmes, W. C., \& Slap, G. B. (1998). Sexual abuse of boys. Definition, prevalence, correlates sequelae, and management. Journal of the American Medical Association, 280(21) $1855-1862$

*Jumaian, A. (2001). Prevalence and long-term impact of child sexual abuse among a sample of male college students in Jordan. Eastern Mediterranean Health Journal, 7(3) $435-440$.

Jumper, S. A. (1995). A meta-analysis of the relationship of child sexual abuse to adult psychological adjustment. Child Abuse \& Neglect, 19(6), 715-728.

Kendall-Tackett, K. A., Meyer Williams L., \& Finkelhor, D. (1993). Impact of sexual abuse on children: A review and synthesis of recent empirical studies. Psychological Bulletin, 113(1), 164-180

*Kenny, M. C., \& McEachern, A. G. (2000). Prevalence and characteristics of childhood sexual abuse in multiethnic female college students. Journal of Child Sexual Abuse, 9(2), 57-70.

*Kercher, G. A., \& McShane, M. (1984). The prevalence of child sexual abuse victimization in an adult sample of Texas residents. Child Abuse \& Neglect, 8, 495-501.

Klonsky, E., \& Moyer, A. (2008). Childhood sexual abuse and non-suicidal self-injury: Meta-analysis. British Journal of Psychiatry, 192(3), 166-170.
Koss, M. P. (1993). Detecting the scope of rape: A review of prevalence research methods. Journal of Interpersonal Violence, 8, 198-222.

*Krugman, S., Mata, L., \& Krugman, R. (1992). Sexual abuse and corporal punishment during childhood: A pilot retrospective survey of University students in Costa Rica. Pediatrics, 90(1), 157-161.

Lalor, K. (2004). Child sexual abuse in Tanzania and Kenya. Child Abuse E' Neglect, $28,833-844$.

Larsen, H. B., \& Helweg-Larsen, K. (2003). Childhood sexual abuse and psychological disturbance in a national youth sample. Nordisk Psykologi, 55(2), 79-93.

Leventhal, J. M. (1998). Epidemiology of sexual abuse of children: Old problems, new directions. Child Abuse \& Neglect, 22(6), 481-491.

*Levett, A. (1989). A study of childhood sexual abuse among South African university women students. South African Journal of Psychology, 19(3), 122-129.

*Lodico, M. A., Gruber, E., \& DiClemente, R. J. (1996). Childhood sexual abuse and coercive sex among school-based adolescents in a Midwestern state. Journal of Adolescent Health, 18, 211-217.

*López, F., Carpintero, E., Hernández, A., Martín, M. J., \& Fuertes, A. (1995). Prevalencia y consecuencias del abuso sexual al menor en España. Child Abuse E Neglect, 19(9), $1039-1050$.

MacMillan, H. L. (1998). Child abuse: A community problem. Canadian Medical Association Journal, 158, 1301-1302.

*MacMillan, H. L, Fleming J. E. Trocmé, N., Boyle, M. H., Wong M., Racine, Y. A et al. (1997). Prevalence of child physical and sexual abuse in the community: Results from the Ontario Health Supplement. JAMA, 278, 131-135.

*Madu, S. N., \& Peltzer, K. (2001). Prevalence and patterns of child sexual abuse and victimperpetrator relationship among secondary school students in the Northern Province (South Africa). Archives of Sexual Behavior, 30(3), 311-321.

*May-Chahal, C., \& Cawson, P. (2005). Measuring child maltreatment in the United Kingdom: A study of the prevalence of child abuse and neglect. Child Abuse \&' Neglect, 29, 969-984.

*Mazza, D., Dennerstein, L., \& Ryan, V. (1996). Physical, sexual and emotional violence against women: A general practice-based prevalence study. Medical Journal of Australia, 164, 14-17.

*McCrann, D., Lalor, K., \& Katabaro, J. K. (2006). Childhood sexual abuse among university students in Tanzania. Child Abuse E' Neglect, 30, 1343-1351.

McGowan, L. P. A., Clark-Carter, D. D., \& Pitts, M. K. (1998). Chronic pelvic pain: A metaanalytic review. Psychology \& Health, 13(5), 937-951.

McMillen, C., \& Zuravin, S. (1998). Social support, therapy and perceived changes in women's attributions for their child sexual abuse. Journal of Child Sexual Abuse, 7(2), 1-15.

Meewise, M. -L., Reitsma, J. B., De Vries, G. -J., Gersons, B. P. R., \& Olff, M. (2007). Cortisol and post-traumatic stress disorder in adults: Systematic review and meta-analysis. British Journal of Psychiatry, 191(4), 367-392.

*Mullen, P. E. Romans-Clarkson, S. E., Walton, V. A., \& Herbison, G. P. (1988, April 16). Impact of sexual and physical abuse on women's mental health. The Lancet, 841-845.

*Nelson, D. E., Higginson, G. K., \& Grant-Worley, J. A. (1994). Using the Youth Risk Behavior Survey to estimate prevalence of sexual abuse among Oregon high school students. The Journal of School Health, 64(10), 413-416.

Neumann, D. A., Houskamp, B. M., Pollock, V. E., \& Briere, J. (1996). The long-term sequelae of childhood sexual abuse in women: A meta-analytic review. Child Maltreatment, 1(1), 6-16.

*Niederberger, J. M. (2002). The perpetrator's strategy as a crucial variable: a representative study of sexual abuse of girls and its sequelae in Switzerland. Child Abuse E' Neglect, 26, $55-71$.

*Oaksford, K. L., \& Frude, N. (2001). The prevalence and nature of child sexual abuse: Evidence from a female university sample in the UK. Child Abuse Review, 10, 49-59.

Oates, R. K., Jones, D. P. H., Denson, D., Sirotnak, A., Gary, N., \& Krugman, R. D. (2000). Erroneous concerns about child sexual abuse. Child Abuse \& Neglect, 24(1), 149-157.

Oddone Paolucci, E., Genuis, M. L., \& Violato, C. (2001). A meta-analysis of the published research on the effects of child sexual abuse. The Journal of Psychology, 135(1), $17-36$

Peleikis, D. E., \& Dahl, A. A. (2005). A systematic review of empirical studies of psychotehrapy with women who were sexually abused as children. Psychotherapy Research, 15(3), $304-315$.

*Pereda, N., \& Forns, M. (2007). Prevalencia y características del abuso sexual infantil en estudiantes universitarios españoles. Child Abuse \&' Neglect, 31, 417-426.

Pereda, N., Guilera, G., Forns, M., \& Gómez-Benito, J. (in press). The international epidemiology of child sexual abuse: twelve years later. Child Abuse \& Neglect.

Pilkington, B., \& Kremer, J. (1995). A review of the epidemiological research on child sexual abuse. Community and college student samples. Child Abuse Review, 4, $84-98$.

Pope, H. G., \& Hudson, J. I. (1995). Can memories of childhood abuse be repressed? Psychological Medicine, 25, 121-126.

Prescott, A., Bank, L., Reid, J. B., Knutson, J. F., Burraston, B. O., \& Eddy, J. M. (2000). The veridicality of punitive childhood experiences reported by adolescents and young adults. Child Abuse \& Neglect, 24(3), 411-423.

*Priest, R. (1992). Child sexual abuse histories among African-American college students: A preliminary study. American Journal of Orthopsychiatry, 62(3), 475-476.

Putnam, F. (2003). Ten-year research update review: Child sexual abuse. Journal of the American Academy of Child and Adolescent Psychiatry, 42(3), 269-278.

Reeker, J., Ensing, D., \& Elliot, R. (1997). A meta-analytic investigation of group treatment outcomes for sexually abused children. Child Abuse \& Neglect, 21(7), 669-680.

*Rew, L., Esparza, D., \& Sands, D. (1991). A comparative study among college students of sexual abuse in childhood. Archives of Psychiatric Nursing, V(6), 331-340.

Rind, B., \& Tromovitch, P. (1997). A meta-analytic review of findings from national samples on psychological correlates of child sexual abuse. Journal of Sex Research, 34(3), 237-255. 
Rind, B., Tromovitch, P., \& Bauserman, R. (1998). A meta-analytic examination of assumed properties of child sexual abuse using college samples. Psychological Bulletin, 124(1), 22-53.

Rispens, J., Aleman, A., \& Goudena, P.P. (1997). Prevention of child sexual abuse victimization: A meta-analysis of school programs. Child Abuse \& Neglect, 21(10), 975-987.

*Robin, R. W., Chester, B., Rasmussen, J. K., Jaranson, J. M., \& Goldman, D. (1997). Prevalence, characteristics, and impact of childhood sexual abuse in a southwestern American Indian tribe. Child Abuse \& Neglect, 21(8), 769-787.

Romano, E., \& De Luca, R. V. (2001). Male sexual abuse: A review of effects, abuse characteristics, and links with later psychological functioning. Aggression and Violent Behavior, 6, 55-78.

*Romero, G. J., Wyatt, G. E., Loeb, T. B., Carmona, J. V., \& Solis, B. M. (1999). The prevalence and circumstances of child sexual abuse among Latina women. Hispanic Journal of Behavioral Sciences, 21(3), 351-365.

Roodman, A. A., \& Clum, G. A. (2001). Revictimization rates and method variance: A metaanalysis. Clinical Psychological Review, 21(2), 183-204.

*Ross, C. A., Keyes, B. B., Xiao, Z., Yan, H., Wang, Z., Zou, Z., et al. (2005). Childhood physical and sexual abuse in China. Journal of Child Sexual Abuse, 14(4), 115-126.

Runyan, D. K. (1998). Prevalence, risk, sensitivity and specificity: A commentary on the epidemiology of child sexual abuse and the development of a research agenda. Child Abuse \& Neglect, 22(6), 493-498.

*Russell, D. E. H. (1983). The incidence and prevalence of intrafamilial and extrafamilial sexual abuse of female children. Child Abuse \& Neglect, 7, 133-146.

*Sariola, H., \& Uutela, A. (1994). The prevalence of child sexual abuse in Finland. Child Abuse \& Neglect, 18(10), 827-835.

*Sariola, H., \& Uutela, A. (1996). The prevalence and context of incest abuse in Finland. Child Abuse \& Neglect, 20(9), 843-850.

*Schei, B. (1990). Prevalence of sexual abuse history in a random sample of Norwegian women. Scandinavian Journal of Social Medicine, 18, 63-68.

*Schein, M., Biderman, A., Baras, M., Bennett, L., Bisharat, B., Borkan, J., et al. (2000). The prevalence of a history of child sexual abuse among adults visiting family practitioners in Israel. Child Abuse \&' Neglect, 24(5), 667-675.

Sharpe, D., \& Faye, C. (2006). Non-epileptic seizures and child sexual abuse: A critical review of the literature. Clinical Psychology Review, 26(8), 1020-1040.

*Siegel, J. M., Sorenson, S. B., Golding, J. M., Burnam, M. A., \& Stein, J. A. (1987). The prevalence of childhood sexual assault. American Journal of Epidemiology, 126(6), 1141-1153.

*Singh, A., Yiing, W. W., \& Nurani, N. K. (1996). Prevalence of childhood sexual abuse among Malaysian paramedical students. Child Abuse \& Neglect, 20(6), $487-492$.
Smolak, L., \& Murnen, S. K. (2002). A meta-analytic examination of the relationship between child sexual abuse and eating disorders. International Journal of Eating Disorders, 31(2), 136-150.

*Sorrenti-Little, L., Bagley, C., \& Robertson, S. (1984). An operational definition of the long-term harmfulness of sexual relations with peers and adults by young children. Journal of the Canadian Association for Young Children, 9, 46-57.

SPSS Inc. (2006). SPSS for Windows, version 15.0.1.Illinois, USA: SPSS Inc. 1986-2006.

Starr, R. H., Dubowitz, H., \& Bush, B. A. (1990). The epidemiology of child maltreatment In R. T. Ammerman \& M. Hersen (Eds.), Children at risk: An evaluation of factors contributing to child abuse and neglect New York, NY: Plenum Press.

*Tang, C. S. (2002). Childhood experiences of sexual abuse among Hong Kong Chinese college students. Child Abuse E Neglect, 26, 23-37.

Tolin, D. F., \& Foa, E. B. (2006). Sex differences in trauma and posttraumatic stress disorder: A quantitative review of 25 years of research. Psychological Bulletin, 132 (6), 959-992.

*Tschumper, A., Narring, F., Meier, C., \& Michaud, P. A. (1998). Sexual victimization in adolescent girls (age 15-20 years) enrolled in post-mandatory schools of professional training programmes in Switzerland. Acta Paediatrica, 87, 212-217.

Violato, C., \& Genuis, M. (1993). Problems of research in male child sexual abuse: A review. Journal of Child Sexual Abuse, 2(3), 33-54.

*Vogeltanz, N. D., Wilsnack, S. C., Harris, T. R., Wilsnack, R. W., Wonderlich, S. A., \& Kristjanson, A. F. (1999). Prevalence and risk factors for childhood sexual abuse in women: National survey findings. Child Abuse E Neglect, 23(6), 579-592.

Walker, C. E., Bonner, B. L., \& Kaufman, K. L. (1988). The physically and sexually abused child. Evaluation and treatment. London, G.B.: Pergamon Press.

West, M. M. (1998). Meta-analysis of studies assessing the efficacy of projective techniques in discriminating child sexual abuse. Child Abuse E Neglect, 22(11), 1151-1166.

Widom, C. S., \& Morris, S. (1997). Accuracy of adult recollections of childhood victimization, Part 2. Childhood sexual abuse. Psychological Assessment, 9(1), 34-46.

World Economic Outlook (2007, April). World economic outlook: Spillovers and cycles in the global economy. Washington DC: International Monetary Fund.

*Wyatt, G. E. (1985). The sexual abuse of Afro-American and White-American women in childhood. Child Abuse \& Neglect, 9, 507-519.

*Wyatt, G. E., Loeb, T. B., Solis, B., Carmona, J. V., \& Romero, G. (1999). The prevalence and circumstances of child sexual abuse: Changes across a decade. Child Abuse \& Neglect 23(1), 45-60.

Wynkoop, T. F., Capps, S. C., \& Priest, B. J. (1995). Incidence and prevalence of child sexual abuse: A critical review of data collection procedures. Journal of Child Sexua Abuse, 4(2), 49-67. 